\title{
Relative impact of model quality and ensemble deficiencies on the performance of ensemble based probabilistic forecasts evaluated through the Brier score
}

\author{
F. Atger \\ Meteo-France (DPREVI), 42, av. G. Coriolis, 31057 Toulouse cedex, France
}

Received: 24 November 2003 - Revised: 30 June 2004 - Accepted: 24 July 2004 - Published: 14 September 2004

\begin{abstract}
The relative impact of model quality and ensemble deficiencies, on the performance of ensemble based probabilistic forecasts, is investigated from a set of idealized experiments. Data are generated according to a statistical model, the validation of which is achieved by comparing generated data to ECMWF ensemble forecasts and analyses. The performance of probabilistic forecasts is evaluated through the reliability and resolution terms of the Brier score. Results are as follows. (i) Resolution appears essentially attributable to the average level of forecast skill. (ii) The lack of reliability comes primarily from forecast bias, and to a lower extent from the ensemble being systematically under-dispersive (or over-dispersive). (iii) Forecast skill contributes very little to reliability in the absence of forecast bias, and this impact is entirely due to the finiteness of the ensemble population. (iv) In the presence of forecast bias, reducing forecast skill leads to improve the reliability. This unexpected feature comes from the fact that lower forecast skill leads to a larger ensemble spread, that compensates for the strong proportion of outliers consequent to forecast bias. (v) The lack of ensemble skill, i.e. non systematic errors affecting both ensemble mean and ensemble spread, contributes little, but significantly, to the lack of reliability and resolution.
\end{abstract}

\section{Introduction}

The validation of operational ensemble prediction systems (EPS) has become an important field of research during the past few years (e.g. Zhu et al., 2001 and references therein). Among other objectives, validation aims at pointing the advantages and drawbacks of the scientific options that have been adopted for the different aspects of the development of an operational EPS: method for the selection of initial perturbations, number of ensemble members, choice of a "stochastic" physics vs. the multi-model approach, etc. Therefore,

Correspondence to: F. Atger

(frederic.atger@meteo.fr) validation often requires a comparison of the performance of ensembles run in meteorological centres where the research strategy differs significantly (e.g. Mullen and Buizza, 2001). This is the case for example when comparing the performance of the two operational ensembles that have been run since the early 1990's, at the U.S. National Centers for Environmental Prediction (NCEP) (Tracton and Kalnay 1993), and at the European Centre for Medium-range Weather Forecasts (ECMWF) (Palmer et al., 1993).

On the other hand, operational ensembles run by different centres are generally based on different forecasting systems, i.e. numerical models and assimilation systems (including the way available observations are collected and selected) that differ sufficiently for giving different forecasts in a given situation. The performance of these forecasting systems is likely to differ, so that the results of a comparison between two EPS may not reflect solely the differences related to the strategy that has been followed for designing the ensemble. The performance of the underlying forecasting system obviously contributes to the overall performance of an ensemble. Normalization of the results allows to compensate for this effect to a certain extent, e.g. when computing a skill-score with respect to a control forecast (Atger, 1999). However, interpretation of such normalized results may be problematic if the relative impact of the quality of the forecasting system, compared to deficiencies intrinsically related to the way the EPS has been designed, remains unknown.

A large variety of verification scores have been proposed for evaluating the quality of an ensemble. Among these scores, the most widely used is the Brier score (Brier, 1950), designed for quantifying the performance of a probabilistic forecast of a dichotomous event. The Brier score is simply the mean square error of forecast probabilities. The decomposition of the Brier score proposed by Murphy (1973) leads to two terms that represent the main attributes of the quality of a probabilistic forecast. Reliability is the first attribute, that quantifies the correspondence between a given probability $p$, and the observed frequency of an event that has been forecast with the probability $p$. More generally, 


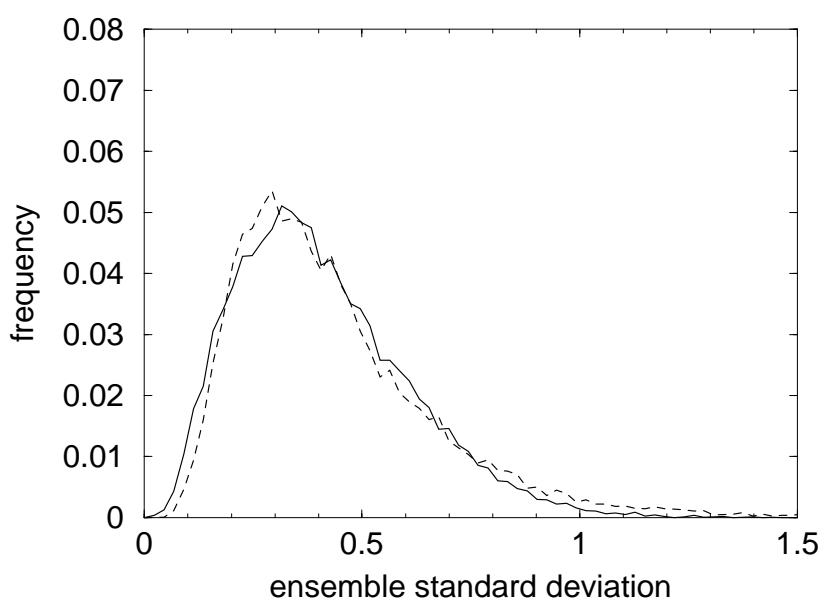

Fig. 1. Distribution of the +96-hour ECMWF ensemble standard deviation, sampled with 67 intervals (solid line), fitted with a transformation of the form $\sigma=\alpha \exp [\beta N(0,1)]$ with $\alpha=0.4$ and $\beta=0.5$ (dashed line).

reliability indicates to which extent a given ensemble distribution proves close to the conditional pdf (probability density function) of the future state of the atmosphere. An estimate of the latter is given by the distribution of the atmospherical states that are observed when a given ensemble distribution is forecast. The second attribute is the resolution, that quantifies the variability of the observed frequency of an event, when the forecast probability of this event varies. In a more general sense, resolution indicates the variability of the conditional pdf, sampled by the observations, when the ensemble distribution varies.

The primary goal of the work presented in this article is to determine to which extent the performance of ensemble based probabilistic forecasts is conditioned by the quality of the underlying forecasting system, i.e. the atmospheric model and the assimilation system. The impact of certain aspects of the quality of an EPS, that are assumed independent of the quality of the forecasting system, is investigated too. Idealized experiments have been designed in order to evaluate the impact of these different factors. The performance of probabilistic forecasts is quantified through the computation of the reliability and resolution terms of the Brier score.

The article is organized as follows. The methodology is exposed in Sect. 2. Results are exposed in Sect. 3, discussed in Sect. 4, summarized in Sect. 5.

\section{Data and methodology}

\subsection{Data}

Data for the idealized experiments have been generated according to a statistical model described in Sect. 2.3. The relevance of this statistical model has been tested by comparing the generated data to analyses and forecasts extracted from the ECMWF archive. Ensemble forecasts and analyses of the

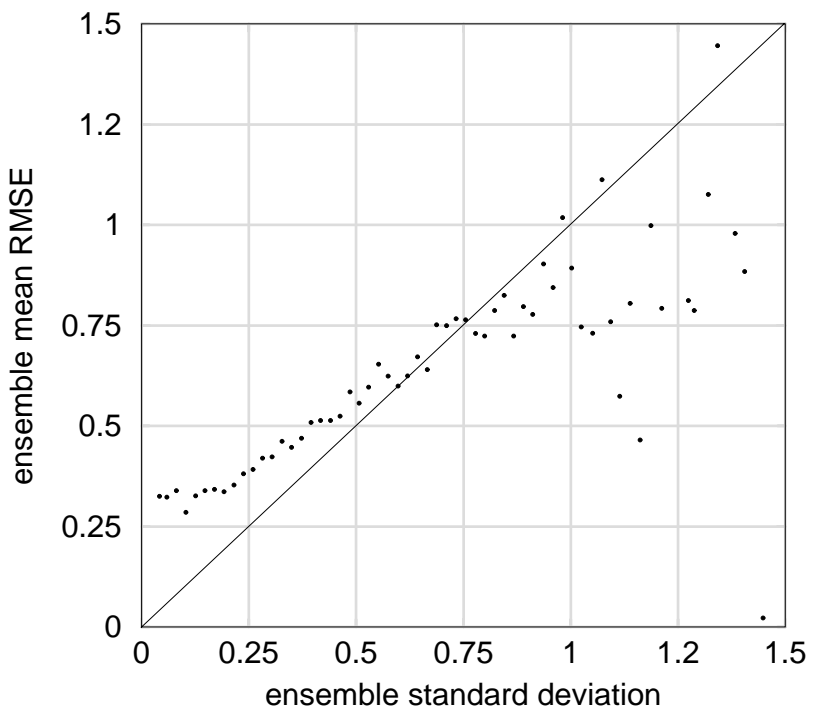

Fig. 2. Correspondence between the +96-hour ECMWF ensemble standard deviation (abcissa) and the ensemble mean RMSE (ordinate). Data have been stratified into 61 classes according to the EPS standard deviation. The average standard deviation in each class is plotted against the RMSE of the ensemble mean computed from the cases found in the class.

850 -hPa temperature anomaly at 48 grid points over Europe $\left.\left.\left.\left(35^{\circ}\right) \mathrm{N}, 60^{\circ}\right) \mathrm{N}, 10^{\circ}\right) \mathrm{W}, 25^{\circ}\right) \mathrm{E}, 5 \times 5^{\circ}$ ) have been retrieved for a period of 4 consecutive winter seasons (December to February), from 10 December 1996 to 28 February 2000, i.e. 351 days. Sample size is $351 \times 48=16848$ cases, considered in this idealized study as independent realizations of a unique random variable. During the considered period the operational version of the ECMWF EPS has been improved several times, as well as the atmospheric model on which it is based, but the horizontal resolution remained the same $\left(T_{L} 159\right)$, as well as the number of ensemble members ( $N=50$ perturbed integrations +1 control integration). Analyses come from the ECMWF high resolution model that was operational during the same period $\left(T_{L} 319\right)$. The climate reference, for the definition of anomalies, has been computed from the ECMWF 15-year reanalysis (Gibson et al. 1997). All data have been standardized with respect to the local analysis standard deviation.

\subsection{Verification}

The performance of ensemble based probabilistic forecasts has been estimated through reliability diagrams and the computation of the Brier score. Unless otherwise stated, the considered event is a positive deviation above 1 standard deviation from the origin.

The reliability curve indicates the correspondence between a given probability, and the observed frequency when this probability is forecast. It is convenient to plot the reliability curve together with an histogram showing the distribution of forecast probabilities. Examples of such reliability diagrams are shown in Fig. 5. 


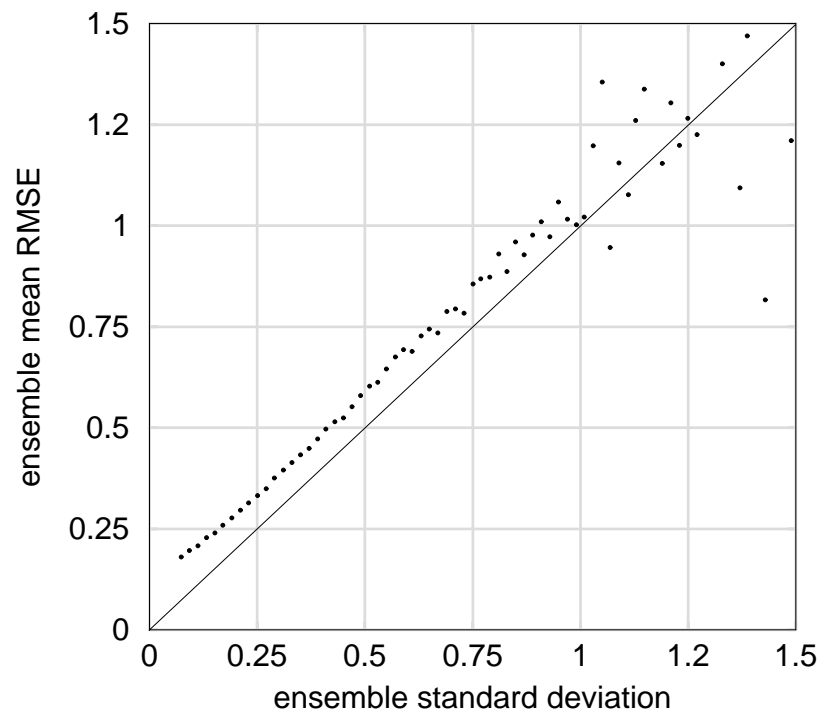

Fig. 3. Same as Fig. 2, but from generated data (10 0000 cases). T he generated ensemble is perfect with respect to any other aspect than under-dispersion $(f s=0.5, f b=-0.16, s b=0.9, s v=0.2$, ems $=0$, ess $=0$ ).

The Brier score $(B S)$ is defined for a dichotomous event as the mean square error of the probability forecast:

$B S=\frac{1}{M} \sum_{i=1}^{M}\left(p_{i}-o_{i}\right)^{2}$,

where $M$ is the number of cases, $p_{i}$ is the forecast probability, $o_{i}$ is the verifying observation $\left(o_{i}=1\right.$ if the event occurs, $o_{i}=0$ if it does not) (Brier 1950). The Brier score is traditionally transformed into a decomposition of 3 terms, initially proposed by Murphy (1973):

$B S=\sum_{k=1}^{T} \frac{m_{k}}{M}\left(p_{k}-o_{k}\right)^{2}-\sum_{k=1}^{T} \frac{m_{k}}{M}\left(o_{k}-o\right)^{2}+o(1-o)$,

when the sample has been divided into $T$ categories, each comprising $m_{k}$ cases when the probability $p_{k}$ is forecast. $o_{k}$ is the observed frequency of the considered event when $p_{k}$ is forecast. $o$ is the observed frequency in the whole sample.

The first part of the decomposition is the reliability term, i.e. the integration, over the whole range of forecast probabilities, of the square difference between the probability and the observed frequency of the event. The reliability term of the Brier score can be seen graphically as the weighted, squared distance between the reliability curve and the $45^{\circ}$ line. It indicates to which extent the forecast probability is calibrated with respect to the a posteriori observed frequency of the considered event.

The second part of the decomposition is the resolution term, i.e. the variance, over the range of forecast probabilities, of the observed frequency of the event. The resolution term of the Brier score can be seen graphically as the weighted, squared distance between the reliability curve

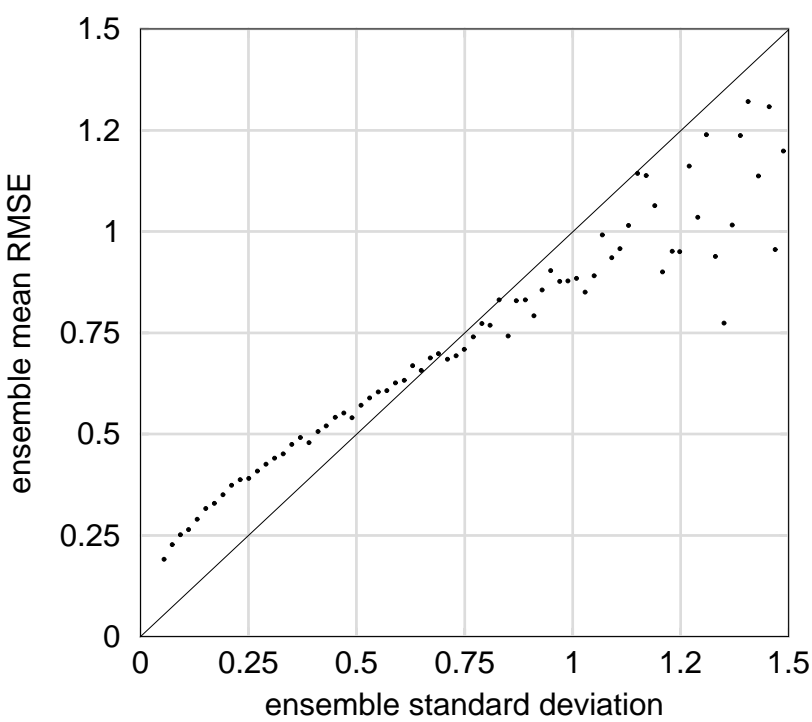

Fig. 4. Same as Fig. 3, but non systematic ensemble deficiencies are considered $(f s=0.5, f b=-0.16, s b=0.9, s v=0.2$, ems $=0.1$, ess $=0.5)$.

and the horizontal line indicating the sample frequency of the considered event. It indicates to which extent the forecast probability discriminates between occurence and nonoccurence of the considered event.

The third part of the decomposition is the uncertainty term, i.e. the variance of the observations, that does not depend on the forecast system but rather reflects the intrinsic difficulty in forecasting the observations.

The resolution term is bounded by the uncertainty term, so that it is convenient to compute the standardized resolution as the ratio of the 2 terms, i.e. $\frac{1}{o(1-o)} \sum_{k=1}^{T} \frac{m_{k}}{M}\left(o_{k}-o\right)^{2}$. This ratio takes its maximum value (Eq. 1) in the case of a perfect deterministic forecast, and more generally when only two probability categories are forecast, leading to observed frequencies 0 and 1 . On the other hand the resolution term equals 0 when the observed frequency of the event is the same whatever the forecast probability.

Note that the reliability term is negatively oriented, as the Brier score (the lower the better) while the resolution term is positively oriented.

\subsection{Statistical model}

\subsubsection{Assumptions and definitions}

Observations are perfectly representative of the truth, the distribution of which is normal $N(0,1)$. The forecasting system under consideration consists in an imperfect model of the truth, run from an imperfect reference initial state. Ensembles members are integrations of the same model, from initial states that differ slightly from the reference, so that they are all consistent with the available observations. The 


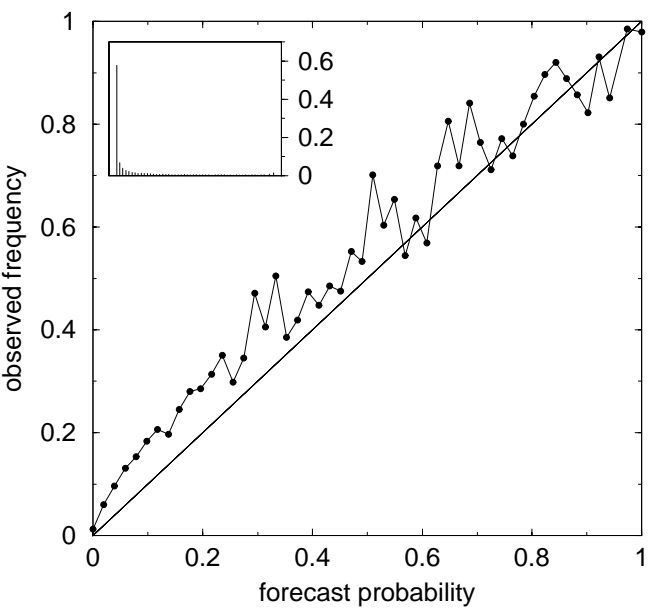

(a)

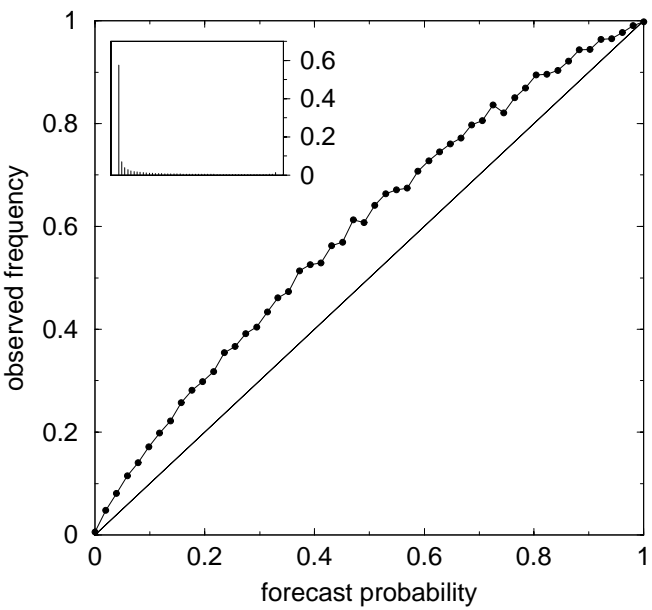

(c)

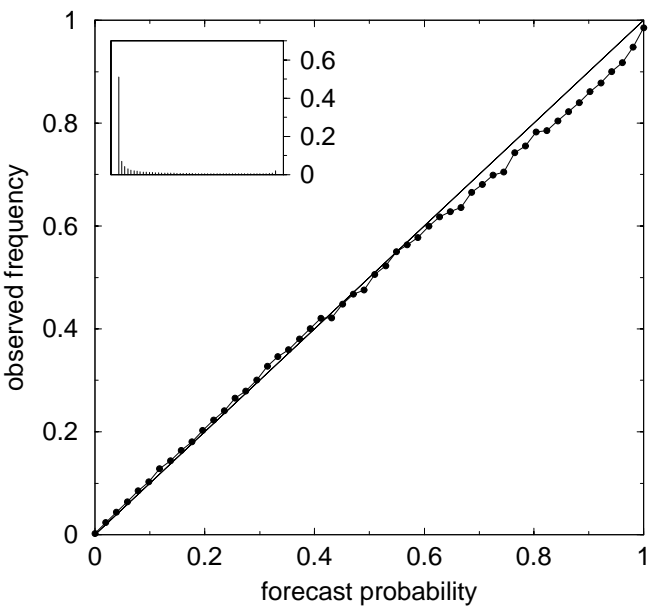

(e)

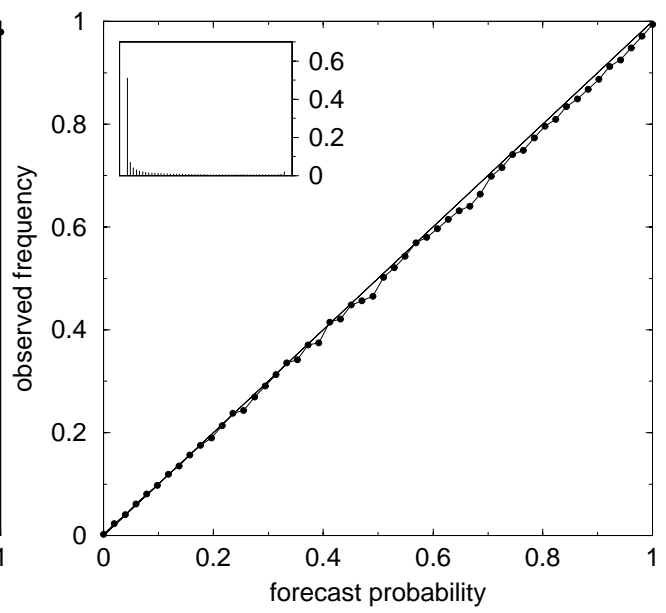

(b)

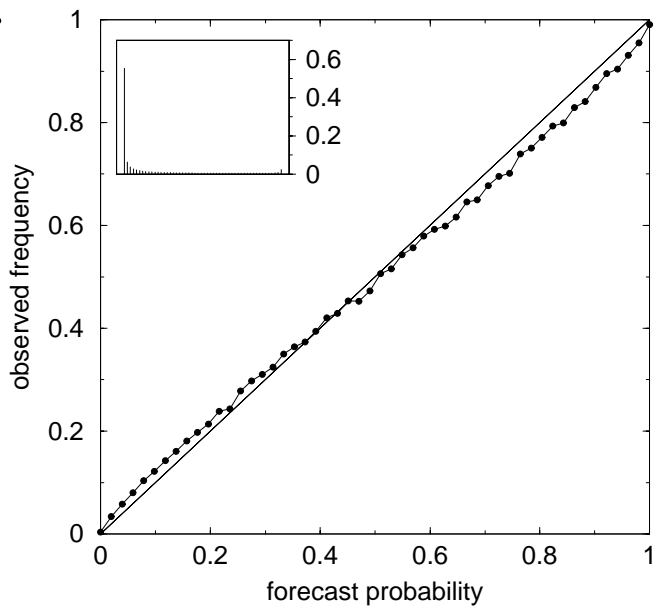

(d)

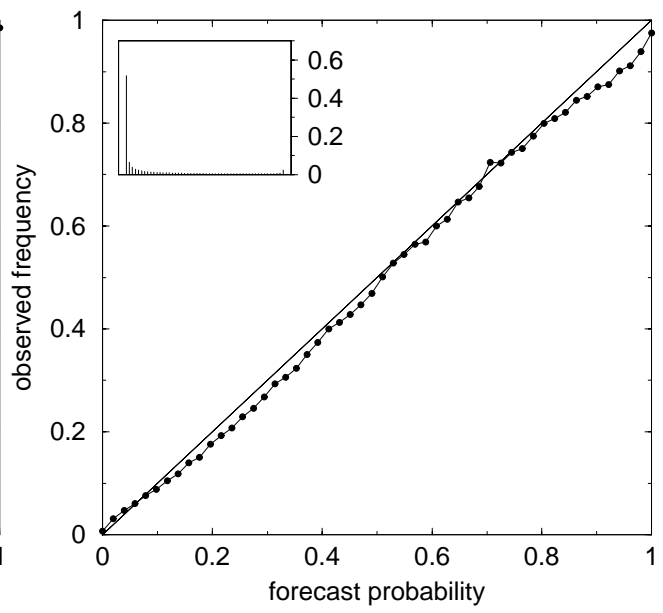

(f)

Fig. 5. Reliability diagrams. The main curve indicates the correspondence between a given forecast probability (abcissa) and the observed frequency of the event when this probability is forecast (ordinate). The histogram shows the distribution of forecast probabilities. The event is a positive deviation of 1 standard deviation from the origin. (a) Probability based on +96-hour ECMWF ensemble forecasts (16848 cases). (b) to (f) Probability based on generated data ( 1 million cases). Forecast skill is set to $f s=0.5$, skill variability is set to $s v=0.2$. (b) No forecast bias, perfect ensemble (no spread bias, ems $=e s s=0$ ). (c) Effect of forecast bias: $f b=-0.16$, perfect ensemble. (d) Effect of spread bias: no forecast bias, $s b=0.9$, perfect ensemble otherwise (ems=ess=0). (e) Effect of a strong lack of ensemble mean skill: no forecast bias, no spread bias, ems =0.2, ess=0. (f) Effect of a strong lack of ensemble spread skill: no forecast bias, no spread bias, ems=0, ess=0.6. (g) Same as (b) to (f) but based on 16848 cases only, and with an arbitrary set of parameters: $f b=-0.16, s b=0.9$, ems $=0.1$, ess $=0.5$. 
forecast bias of ensemble members is thus identical to that of the reference run (i.e. the integration from the reference initial state). Ensemble members are normally distributed. Unless otherwise stated, the number of ensemble members is fixed to 51 (in order to facilitate comparisons to the ECMWF ensemble).

\subsubsection{Perfect ensemble}

In this section the forecasting system is assumed not to be biased, i.e. the mean error of the reference run is zero, as well as that of any ensemble member. The perfect ensemble is defined as an idealized, perfectly reliable ensemble, whose members are assumed to be drawn from $N\left(m_{p}, s_{p}\right)$, according to the normal assumption mentioned above. Since there is no forecast bias, the perfect ensemble mean $m_{p}$ is a draw from the truth distribution, i.e. $N(0,1)$. The perfect ensemble standard deviation $s_{p}$ is arbitrarily drawn from $\alpha \exp [\beta \mathrm{N}(0$, 1)] (log-normal distribution). Figure 1 shows that this choice is consistent with the actual distribution of the standard deviation of the ECMWF operational ensemble.

Since the perfect ensemble is perfectly reliable, the verifying observation is also drawn from $N\left(m_{p}, s_{p}\right)$. The standard deviation of this distribution indicates the level of uncertainty related to the forecast of the verifying observation (the ensemble being perfect, this uncertainty is indeed perfectly related to the ensemble spread). Therefore, the mean of $s_{p}$ indicates the average level of forecast uncertainty, given the forecasting system under consideration, called forecast skill $(f s)$ in the following. Similarly, the standard deviation of $s_{p}$ indicates the variability of the level of forecast uncertainty, called skill variability $(s v)$ in the following. The parameters $f s$ and $s v$ indicate two characteristics of the forecasting system that are independent of other parameters that might characterize the way an ensemble is designed.

From the above definitions, it comes that $\alpha=\frac{f s^{2}}{\sqrt{f s^{2}+s v^{2}}}$ and $\beta^{2}=\log \left(\frac{s v^{2}}{f s^{2}}+1\right)$.

The average level of forecast skill $(f s)$, as well as the variability of the forecast skill ( $s v)$, are related to the level of atmospheric predictability. However, in the present study, predictability is not seen as an intrinsic property of the atmosphere, but rather reflects the ability of a given forecasting system to predict the evolution of the atmosphere. Although it is obviously constrained by the actual initial conditions, forecast skill is thus an attribute of the quality of a forecasting system. And its variability, highly dependent on the atmosphere dynamics, is still a characteristic of the forecasting system.Generated ensembles Imperfect ensembles are generated from a (potentially) biased forecasting system. Specifically, ensemble members are drawn from a normal distribution $\mathrm{N}\left(m_{e}, s_{e}\right)$ that differs from $N\left(m_{p}, s_{p}\right)$ according to systematic deficiencies of both the forecasting system and the ensemble. First, a systematic forecast bias $(f b)$ is taken into account:

$m_{e}=m_{p}+f b$.

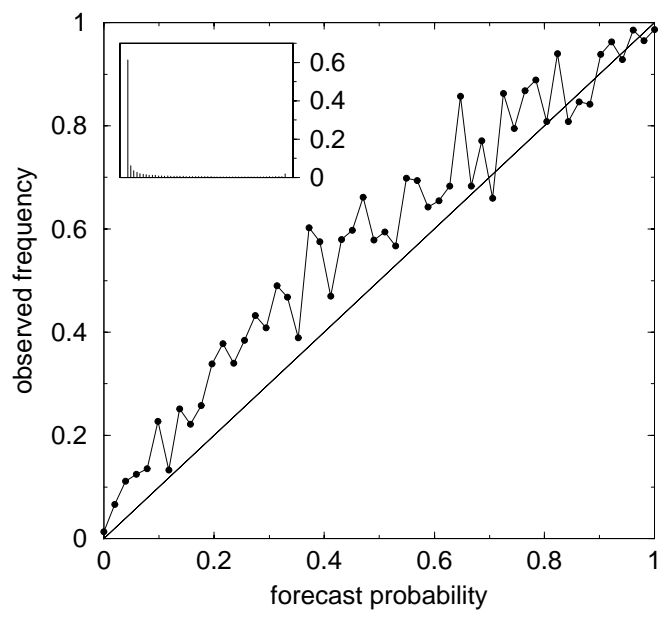

(g)

Fig. 5. Continued.

Secondly, a systematic (negative) spread bias $(s b)$, i.e. a systematic under-dispersion, is taken into account $(0<s b<1$ for the sake of realism, but with no loss of generality):

$s_{e}=s_{p} s b$.

The parameter $f b$ is a characteristic of the forecasting system that is independent on the way the ensemble is designed (under the assumptions given in Sect. 2.3.1), while $s b$ is a characteristic of the ensemble that has no reason to depend on the quality of the forecasting system.

At this stage, the generated ensemble is perfect with respect to any other aspect of the performance than the systematic lack of dispersion. As a consequence the relationship between ensemble spread and forecast skill is virtually perfect, although systematically biased when $s b<1$ (Fig. 3). This is clearly not a realistic feature when compared to an operational ensemble as the ECMWF EPS (Fig. 2). Non systematic aspects of the "intrinsic" quality of the generated ensemble (i.e. independent on the quality of the underlying forecasting system) are taken into account by modifying Eqs. (3) and (4):

$m_{e}=\xi_{m}\left(m_{p}+f b\right)$

$s_{e}=\xi_{s} s_{p} s b$,

where $\xi_{m}$ and $\xi_{s}$ are drawn from a uniform distribution centered in 1. The half-amplitude of the distribution of $\xi_{m}\left(\xi_{s}\right)$ is the parameter ems (ess) standing for ensemble mean skill (ensemble spread skill), that indicates to which extent the ensemble fails to sample the pdf because of non systematic errors affecting the ensemble mean (spread). The parameters ems and ess indicate two intrinsic characteristics of the ensemble and are independent of parameters that characterize the performance of the forecasting system. When ensembles are generated from Eqs. $\left(3^{\prime}\right)$ and $\left(4^{\prime}\right)$ the relationship between spread and skill (Fig. 4) resembles that observed in ECMWF data (Fig. 2). 

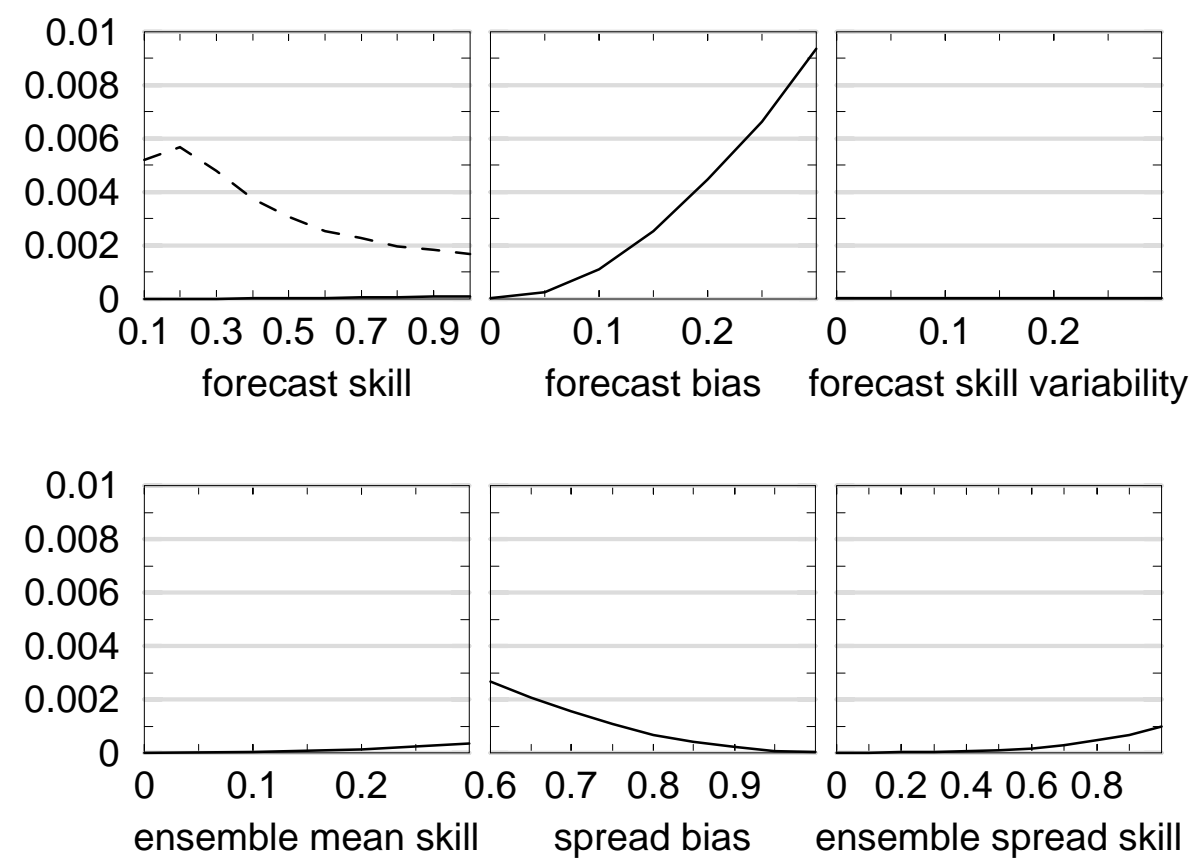

Fig. 6. Reliability term of the Brier score as a function of the different factors. The forecast skill is arbitrarily set to $f s=0.5$, except when it varies. The skill variability is arbitrarily set to $s v=0.2$, except when it varies. The impact of forecast skill is evaluated in the case of a perfect ensemble $(s b=1, e m s=e s s=0)$, with no forecast bias $(f b=0$, solid curve) or in the case of a moderate negative forecast bias $(f b=-0.16$, dashed curve). The impact of forecast bias is evaluated in the case of a perfect ensemble $(s b=1, e m s=e s s=0)$. The impact of skill variability is evaluated in the absence of forecast bias $(f b=0)$ and with a perfect ensemble $(s b=1, e m s=e s s=0)$. The impact of spread bias is evaluated in the absence of forecast bias $(f b=0)$ and with a perfect ensemble skill (ems $=e s s=0)$. The impact of ensemble mean skill and ensemble spread skill is evaluated separately in the absence of forecast bias $(f b=0)$ and with no spread bias $(s b=1)$.

\subsection{Experiments}

6 parameters determine the statistical model that has been used for running the experiments. The 3 parameters $f b$ (forecast bias), $f s$ (forecast skill) and $s v$ (skill variability) indicate the quality of the underlying forecasting system. They are independent of the characteristics of the generated ensemble. The parameters $s b$ (spread bias), ems (ensemble mean skill) and ess (ensemble spread skill) are related to the intrinsic quality of the ensemble, since they are not attributable to the underlying forecasting system.

The method for generating the data, as described above, implies that the modification of certain parameters ( $f s$ and $s v$ ) have an impact on the distribution of observations. In order to get comparable results, all generated data have been standardized with respect to the standard deviation of the generated observations.

ECMWF ensemble forecasts at different lead-times have been compared to generated data in order to determine a realistic range for the different parameters.

The definition of the parameter $f b$ allows a direct estimation, as the algebraic mean of the forecast error. Typical values are $-0.1 /-0.2$ for $+48 /+144$-hour ECMWF forecasts ( -0.16 for +96 -hour).

The parameter $f s$ is assumed to be of the same order as the standard deviation of the forecast error of the EPS con- trol forecast, therefore varying mainly according to the forecast lead-time. Typical values of the error are $0.3 / 0.7$ for $+48 /+144$-hour ECMWF forecasts ( 0.5 for +96 -hour).

Skill variability (parameter $s v$ ) is assumed not to be very different from the day-to-day variability of the operational ensemble standard deviation. Typical values of spread variability are $0.1 / 0.2$ for $+48 /+144$-hour ECMWF forecasts.

There is empirical evidence indicating that $s b$ is moderately negative in operational ensembles, i.e. ensembles generally suffer from a limited under-dispersion (e.g. Buizza, 1997; Toth and Kalnay, 1997).

Parameters ems and ess have been tuned empirically in order to reproduce the characteristics of operational ensembles, in particular the shape of the reliability curve and the relationship between ensemble spread and forecast skill, when other parameters vary.

In the experiments $f b$ ranges from 0 (no forecast bias) to -0.3 (large negative forecast bias). $f s$ ranges from 0.1 (almost perfect forecast, e.g. very short range forecast) to 1 (very poor forecast, e.g. late medium range forecast). $s v$ ranges from 0 (no variability of forecast skill) to 0.3 (high variability of forecast skill). $s b$ ranges from 0.6 (severe underdispersion) to 1 (no spread bias). ems ranges from 0 (no error affecting the ensemble mean, other than systematic forecast bias) to 0.5 (large, non systematic errors affecting the ensemble mean). ess ranges from 0 (no error affecting 

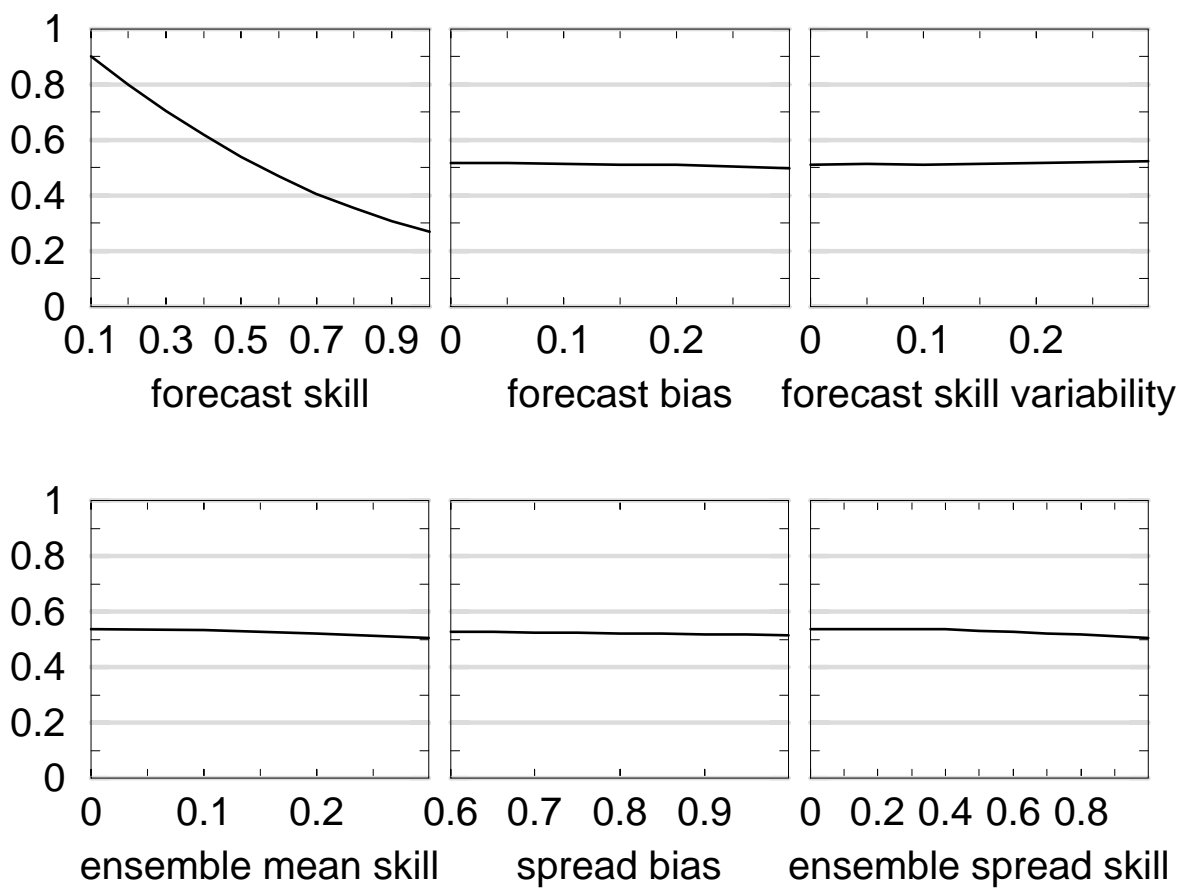

Fig. 7. Same as Fig. 6, but for the resolution term of the Brier score. The effect of a negative forecast bias on the impact of the forecast skill is not shown (undistinguishable).

the ensemble spread, other than the systematic lack of dispersion) to 1 (large, non systematic errors affecting the ensemble spread).

\section{Results}

Unless otherwise stated, one million verification cases have been generated for each experiment. The impact of the different factors is investigated by varying the value of the 6 parameters and evaluating the performance of probabilistic forecasts through reliability curves and the reliability and resolution terms of the Brier score. Qualitatively, forecast skill and skill variability are expected to have an impact on resolution. Forecast bias and spread bias are expected to have an impact on reliability. Ensemble mean skill and ensemble spread skill are expected to have an impact both on reliability and resolution.

\subsection{Reliability curves}

Figure 5 shows the reliability curve obtained from +96 -hour ECMWF ensemble forecasts, as a reference (Fig. 5a), together with those obtained with generated data. Forecast skill and skill variability have been arbitrarily fixed $\left(f_{s}=0.5\right.$, $s v=0.2$ ), since these two parameters are not expected to have any impact on reliability. The other parameters have been set as follows:

(i) No forecast bias $(f b=0)$ and perfect ensemble, i.e. $s b=1$ and $e m s=e s s=0$ (Fig. 5b). Reliability is not perfect, although the observation is drawn from the same distribution as en- semble members. Lower probabilities are slightly underestimated (this effect is hardly visible) while higher probabilities are slightly overestimated. The significance of this feature is discussed in Sect. 4.

(ii) Realistic forecast bias $(f b=-0.16)$ and perfect ensemble (Fig. 5c). The typical effect of a negative bias is similar to that observed for ECMWF data (Fig. 5a). Because the sign of the bias is opposite to that of the considered event (positive deviation larger than 1 standard deviation) the forecast probability is systematically underestimated.

(iii) No forecast bias, realistic spread bias $(s b=0.9)$, perfect ensemble otherwise i.e. ems $=$ ess $=0$ (Fig. $5 \mathrm{~d}$ ). The effect of spread bias is typical too: because of the systematic underdispersion, lower probabilities are underestimated while higher probabilities are overestimated. Combined to that of a negative forecast bias (Fig. 5c) this effect leads to a reliability curve close to that obtained from ECMWF data (Fig. 5a).

(iv) No forecast bias, no spread bias, but a strong lack of skill affects the ensemble mean only (ems $=0.2$, ess $=0$ ) (Fig. 5e). The impact is similar to that of a moderate spread bias: underestimation of lower probabilities, overestimation of higher probabilities.

(v) No forecast bias, no spread bias, but a strong lack of skill affects the ensemble spread only (ems $=0$, ess $=0.6$ ) (Fig. 5f). The typical impact is an overall overestimation of forecast probabilities, of the same amplitude as caused by a moderate spread bias.

The combined effect of the different factors allows to reproduce the main characteristics of the reliability curve obtained from ECMWF data (Fig. 5g). As in the example 
shown in Sect. 2.3 .3 (Fig. 4) the parameters have been set empirically as follows: $f s=0.5, f b=-0.16, s v=0.2, s b=0.9$, ems $=0.1$, ess $=0.5$. In order to get a similar level of "noise", when plotting the reliability curve, as obtained from ECMWF data, the experiment consists here in generating 16848 cases only.

\subsection{Reliability}

Figure 6 shows the impact of the different factors, considered separately, on the reliability term of the Brier score. The lack of reliability comes mainly from the forecast bias, and to a lower extent from the spread bias. The lack of ensemble skill, attributable to non systematic errors affecting both the ensemble mean and the ensemble spread, has a limited impact. There is no impact of the forecast skill variability, as expected. In the absence of forecast bias the impact of forecast skill is very small, as expected, but reliability does improve slightly when the skill increases. This result is discussed in Sect. 4.

When the forecast bias is set to $f b=-0.16$, reliability improves when the skill decreases, i.e. the reliability term of the Brier score is numerically reduced. This improvement is larger when the parameters $f s$ and $f b$ are comparable, i.e. when forecast bias and forecast error have the same amplitude, and it is emphasized when the forecast bias increases (not shown). This rather unexpected feature can be explained by the fact that increasing the forecast skill has the primary effect of decreasing the ensemble spread, provided the relationship between spread and skill exists. When the forecast skill is high, the spread tends to be small and the ensemble distribution is sharp. The shift of the ensemble distribution with respect to the verification, attributable to the forecast bias, leads to a strong proportion of outliers. This induces a systematic underestimation of the forecast probability of an infrequent event (such as the event considered in the present study). When the forecast skill is lower, the spread tends to be large and the ensemble distribution is flatter. The proportion of outliers due to the forecast bias (i.e. related to the shift of the distribution) is thus reduced, so that the underestimation of the forecast probability is limited.

\subsection{Resolution}

Figure 7 shows the impact of the different factors on the resolution term of the Brier score, after standardization by the uncertainty term (Sect. 2.2). The main result is that resolution is almost entirely due to the forecast skill. Ensemble skill (parameters ems and ess) has a definite impact, although small in amplitude. Resolution does increase with the skill variability, but the impact is hardly visible. On the other hand there is a slight decrease of resolution when the forecast bias or the spread bias grows. This last effect is discussed in Sect. 4.

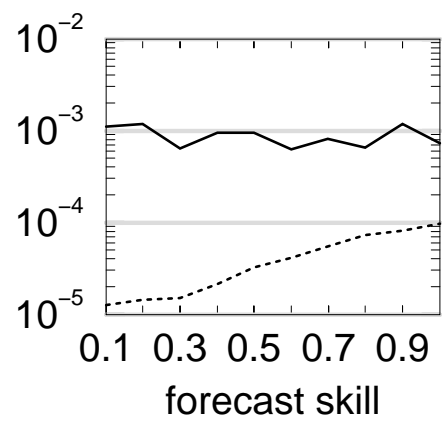

Fig. 8. Same as Fig. 6 (forecast skill panel only, y-coordinate follows a logarithmic scale), but the curves have been obtained by generating 10000 cases (solid line) and 1 million cases (dashed line)

\section{Discussion}

\subsection{Impact of the number of generated cases}

The results presented in the previous section have been obtained by generating a very large number of cases for each experiment (one million). In the real world the number of verification cases that can be considered as independent realizations of the same random variable is rather limited, especially if one wants to take into account space and time correlations (Atger, 2003). A critical issue is thus whether the results presented above have any chance to be confirmed by performance evaluations based on real EPS data.

The relative impact of the different factors has been investigated from 10000 verification cases instead of 1 million. Variations of the reliability term of the Brier score are reduced when the number of cases decreases. In particular, the slight degradation of reliability when the forecast skill decreases (in the absence of forecast bias) cannot be demonstrated with a sample consisting of 10000 cases (Fig. 8). This seems to be due to the fact that this degradation is so tiny that it tends to be of the same order as the noise due to the lack of sampling.

It was mentioned in Sect. 2 that a lack of resolution, in our case almost entirely due to a decrease of the forecast skill, makes the reliability curve closer to the horizontal line that indicates the sample frequency of the event. Assuming a perfect ensemble and no forecast bias, this means that the lack of resolution makes the reliability curve pivot clockwise around the point of correspondence of the average forecast probability with the overall frequency of the event. This effect is visible in Fig. 5b, but it is clear from this figure that a very large sample is needed for this effect to have any significant impact on the reliability term of the Brier score. Figure 9 shows for example the reliability curve obtained in the same configuration as in Fig. 5b, but from a sample of 10000 cases instead of 1 million. Given the level of noise of the curve, it is not surprising that Fig. 8 shows a constant level of reliability when the forecast skill grows from 0.1 to 1 . Note that this level of noise is similar to that shown in Fig. 5a, obtained 


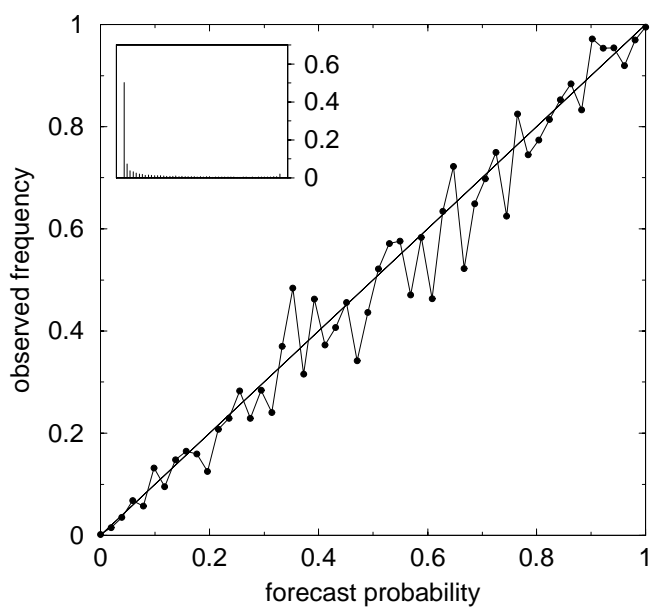

Fig. 9. Same as Fig. 5b but computed from 10000 generated cases instead of 1 million.

from 16848 ECMWF cases accumulated over 4 winter seasons over Europe.

On the contrary, little effect has been found when reducing the size of the sample for evaluating the resolution term of the Brier score. Estimating resolution seems much easier, even from limited samples, than estimating reliability. This can be explained again by graphical considerations, the $45^{\circ}$ line being generally closer to the reliability curve (due to the high level of reliability) than the horizontal line indicating the sample frequency of the event (Atger, 2004).

\subsection{Impact of the number of ensemble members}

For facilitating comparisons with the ECMWF operational EPS, the number of members of the generated ensembles has been set to $N=51$ in the previous section. Increasing or decreasing $N$ may have an effect on the performance of ensemble based probabilistic forecasts, and consequently on the relative impact of the different factors that have been considered.

Richardson (2001) has shown that decreasing the ensemble population results in a numerical increase of the reliability term of the Brier score, at least when the number of verification cases is large. It was mentioned in the previous section how the lack of resolution makes the reliability curve pivot around the point indicating the correspondence between the average forecast probability and the overall frequency of the event, thus increasing the reliability term. Figure 10 shows that this slope effect is emphasized when $N$ is reduced, leading to an increasing overestimation of probabilities above the frequency of the event. This can be seen as an effect of a poorer sampling of the pdf, due to the reduction of the ensemble population. For example, when all the members forecast the event, the probability for the event to occur should not be 1 but "more than $N-1 / N$ " since there is no way to estimate a probability between $N-1 / N$ and 1 . In

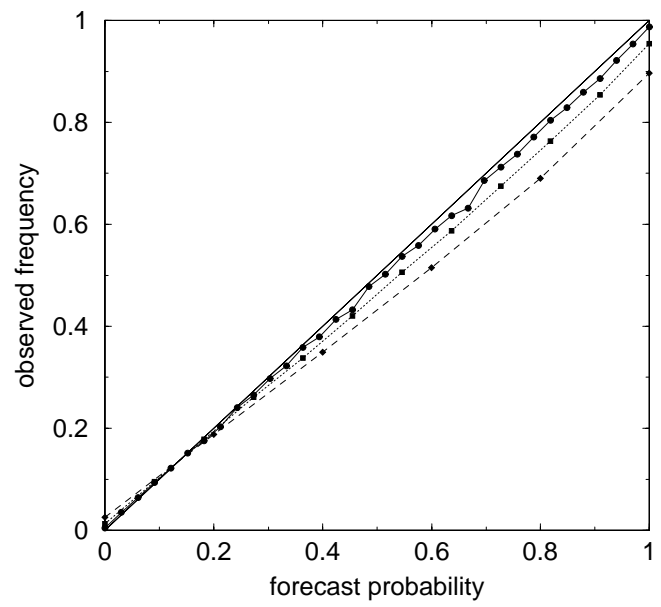

Fig. 10. Same as Fig. $5 b$ but the number of ensemble members is set to 33 (solid line, circles), 11 (dotted line, squares) and 5 (dashed line, diamonds). The distribution of forecast probabilities is not shown.

other words, when $N$ is small, extra ensemble members are missing that could sample the tails of the pdf. Similarly, extra members are missing that could sample the pdf between 2 consecutive existing members.

In fact, one would expect the reliability term of the Brier score to be zero and the reliability curve to be perfectly aligned along the $45^{\circ}$ line in the case of a perfect ensemble. If it is not exactly the case in Fig. 5b, and not at all the case in Fig. 10, it is just because $N$ is finite. This effect is emphasized when the forecast skill decreases (Fig. 11, forecast skill panel): because the uncertainty becomes larger, the number of members that are needed for sampling the pdf increases. When the error is low, even small (perfect) ensembles are able to sample the pdf, while a large number of (perfect) ensemble members is required when the error is high. Because the number of ensemble members is finite, only when the resolution is perfect, i.e. when $f s=0$, the reliability term of the Brier score is zero in the case of a perfect ensemble (Figs. 6 and 11 , forecast skill panel).

The forecast bias panel of Fig. 11 shows that above a certain level of systematic bias (approx. 0.05) the impact of reducing the number of ensemble members is reverse: reliability improves when the population is reduced. This is because the effect of a "negative" forecast bias compensates that of reducing the ensemble population, as shown in Fig. 12a. The former leads to an underestimation of the probability of a "positive" deviation from the origin, while the latter leads to an overestimation of forecast probabilities above the frequency of the considered event, as discussed above. On the contrary, these two effects would cumulate if the sign of the forecast bias was the same as that of the forecast deviation. This might be the case, for example, when evaluating probabilistic precipitation forecasts produced with a model overforecasting precipitation amounts. 

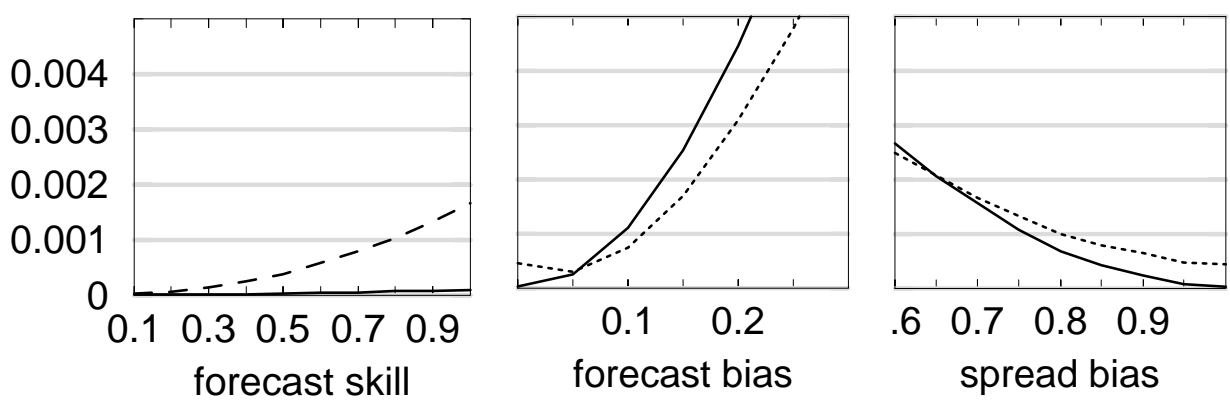

Fig. 11. Same as Fig. 6 (forecast skill, forecast bias and spread bias panels only), but generated ensembles consisting of 11 members (dashed line) and 51 members (solid line).

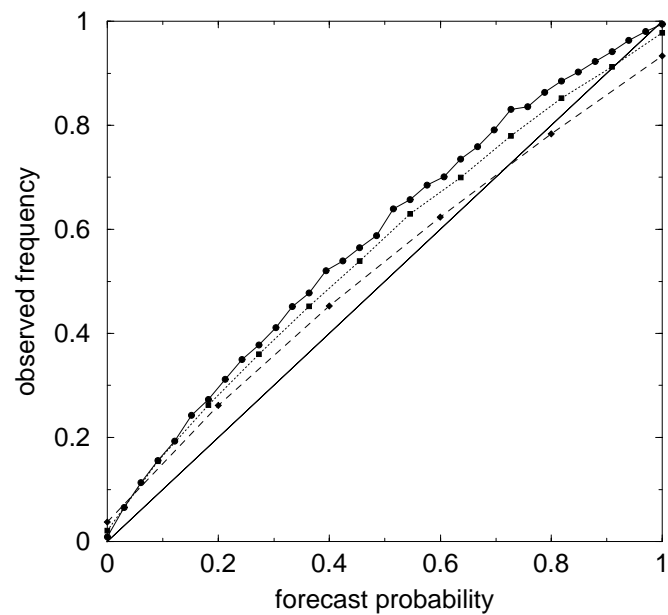

(a)

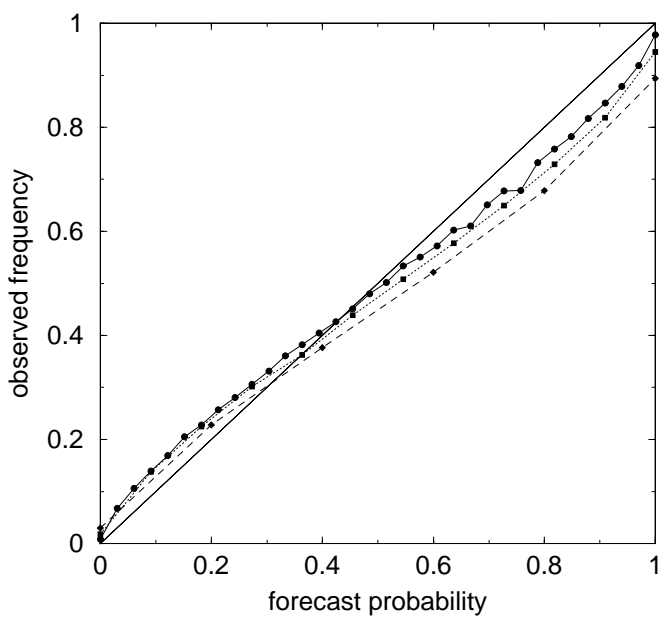

(b)

Fig. 12. Same as Figs. $5 c$ and d, respectively, but the number of ensemble members is set to 33 (solid line, circles), 11 (dotted line, squares) and 5 (dashed line, diamonds). The distribution of forecast probabilities is not shown.

An increase of the spread bias also tends to attenuate the impact on reliability of a reduction of the number of ensemble members (Fig. 11, spread bias panel). Only in the case of a strong spread bias the reliability term of the Brier score increases with the number of ensemble members. Again, this is because the effect of underdispersion compensates that of reducing the number of ensemble members (Fig. 12b). The former leads to an underestimation of forecast probabilities, for an infrequent event, while the latter leads to an overestimation of probabilities above the frequency of the considered event.

Decreasing the ensemble population has only little (negative) effect on resolution. The relative impact of the different factors is unchanged, i.e. the forecast skill explains almost all the variations of the resolution term of the Brier score.

The combined effect of limiting both the number of ensemble members and the number of verification cases is beyond the scope of this paper. This is a crucial issue for the validation of operational ensembles that has been extensively studied by Candille (2003a, b).

\subsection{Impact of the frequency of the forecast event}

All the results presented above have been obtained for an event occurring with an overall frequency close to $16 \%$ (positive deviation above 1 standard deviation). Considering less frequent events (e.g. a positive deviation above 2 standard deviations) has the effect of decreasing the reliability term of the Brier score, especially when the forecast bias and/or the spread bias is high (not shown). However, the relative impact of the different factors is roughly unchanged, except that forecast bias and spread bias contribute at a closer level to the reliability term of the Brier score (not shown).

\section{Summary}

The impact of model quality and ensemble deficiencies on the performance of ensemble based probabilistic forecasts has been investigated from a series of idealized experiments. Data are generated according to a statistical model validated through a comparison with ECMWF ensemble forecasts and analyses. The performance is evaluated from reliability 
curves and the reliability and resolution terms of the Brier score.

6 different factors are considered in the study: forecast bias, forecast skill and skill variability are entirely attributable to the forecasting system (in a wide sense, i.e. the model, the assimilation system and the observations network) and do not depend on the characteristics of the ensemble; spread bias, ensemble mean skill and ensemble spread skill reflect several aspects of the quality of an ensemble that are assumed not to depend directly on the quality of the underlying forecasting system.

The main results are the following:

1) The lack of reliability comes primarily from forecast bias, and to a lower extent from spread bias, i.e. from the ensemble being systematically underdispersive (in general). Ensemble mean skill and ensemble spread skill contributes little to reliability.

2) In the absence of forecast bias, forecast skill contributes very little to reliability. This small impact is entirely due to the fact that a finite number of ensemble members does not allow a perfect sampling of the pdf.

3 In the presence of forecast bias, decreasing the forecast skill leads to an improvement of the reliability. This is because a lower forecast skill leads to a larger ensemble spread that compensates the high proportion of outliers consequent to forecast bias. This impact of the forecast skill on the reliability term of the Brier score is of the same order as the impact of moderate variations of the forecast bias.

4) Resolution is essentially attributable to forecast skill. The impact of skill variability is comparatively very small. There is a little impact on resolution of ensemble mean skill and ensemble spread skill.

Acknowledgements. The author would like to thank G. Candille and O. Talagrand for fruitful discussions. Y. Zhu provided helpful comments on an earlier version of the manuscript.

Edited by: A. Osborne

Reviewed by: G. Candille and Y. Zhu

\section{References}

Atger, F.: The skill of ensemble prediction systems, Mon. Wea. Rev., 127, 9, 1941-1953, 1999.

Atger, F.: Spatial and interannual variability of the reliability of ensemble based probabilistic forecasts: Consequences for calibration, Mon. Wea. Rev., 131, 1509-1523, 2003.

Atger, F.: Estimation of the expected reliability of ensemble based probabilistic forecasts, Quart. J. Roy. Meteor. Soc., 130, 627646, 2004.

Brier, G. W.: Verification of forecasts expressed in terms of probability, Mon. Wea. Rev., 78, 1-3, 1950.

Buizza, R.: Potential forecast skill of ensemble prediction, and spread and skill distributions of the ECMWF Ensemble Prediction System, Mon. Wea. Rev., 125, 99-119, 1997.

Candille, G.: On the limitations to objective evaluation of ensemble prediction systems, Workshop on Ensemble Weather Forecasting in the Short to Medium Range, Val-Morin, Québec, Canada, 1820 September, 2003a.

Candille, G.: Validation des systèmes de prévisions météorologiques probabilistes, $\mathrm{PhD}$ thesis, Université Pierre et Marie Curie, Paris, June, 2003b.

Gibson, J. K., Kallberg, P., Uppala, S., Hernandez, A., Nomura, A., and Serrano, E.: ERA description, ECMWF Re-Analysis Project Report Series, 1, 72, 1997.

Mullen, S. L. and Buizza, R.: Quantitative precipitation forecasts over the United States by the ECMWF Ensemble Prediction System, Mon. Wea. Rev., 129, 638-661, 2001.

Murphy, A. H.: A new vector partition of the probability score, J. Appl. Meteor., 12, 595-600, 1973.

Palmer, T. N., Molteni, F., Mureau, R., Buizza, R., Chapelet, P., and Tribbia, J.: Ensemble prediction. Seminar on Validation of Models over Europe, Reading, U.K., European Centre for Mediumrange Weather Forecasts, Proceedings, 1, 21-66, 1993.

Richardson, D. S.: Measures of skill and value of ensemble prediction systems, their interrelationship and the effect of ensemble size, Quart. J. Roy. Meteor. Soc., 127, 2473-2489, 2001.

Toth, Z. and Kalnay, E.: Ensemble forecasting at NCEP and the breeding method, Mon. Wea. Rev., 125, 3297-3319, 1997.

Tracton, M. S. and Kalnay, E.: Operational ensemble prediction at the National Meteorological Center: practical aspects, Wea. Forecasting, 8, 379-398, 1993.

Zhu, Y., Toth, Z., Wobus, R., Richardson, D., and Mylne, K.: The economic value of ensemble based weather forecasts, Bull. Amer. Meteorol. Soc., 83, 73-83, 2001. 